INRA Prod. Anim.,

2014, 27 (1), 41-48

\section{La place du vétérinaire en milieu de montagne : évolution et futur}

C. $R O Y$

Clinique Vétérinaire des Mazets, F-15400 Riom-es-Montagnes, France

Courriel : roille@orange.fr

Le binôme " vétérinaire-éleveur » constitue le maillon principal de la santé animale en France. Cette collaboration étroite, particulièrement pour l'élevage en montagne, doit faire face aujourd'hui aux mutations du monde agricole et de la profession vétérinaire.

La profession vétérinaire, à l'instar des autres professions de santé, est confrontée à une profonde mutation de sa démographie : les zones rurales de montagne n'attirent plus durablement les jeunes diplômés. Pourtant, si la densité de population dans ces zones est en constante diminution, il n'en est pas de même pour la population animale. Ainsi, le vétérinaire peut y trouver une «patientèle » toujours abondante. Comment la profession vétérinaire a-t-elle évolué depuis la fin du XX ${ }^{\text {ème }}$ siècle dans ces zones? Quel avenir peuvent encore espérer les vétérinaires installés en zones de montagne? Quelles sont les spécificités de l'exercice vétérinaire en montagne qui le rendent tantôt attrayant aux yeux des uns, tantôt repoussant aux yeux des autres?

\section{1 / La démographie vétéri- naire en zone de montagne}

\section{1 / Qu'entend-on par « milieu de montagne" ?}

Les zones de montagne sont définies par la loi Montagne du 9 janvier 1985. Cette loi a pour objectif de fixer des dispositions adaptées aux spécificités des territoires montagnards, notamment en matière agricole, et qui ne s'appliquent qu'à eux. A titre d'exemple, les agriculteurs en zone de montagne bénéficient de l'Indemnité Compensatoire de Handicap Naturel (ICHN). Un zonage juridique détermine ainsi les zones de classement Montagne (carte 1). Cette définition de la montagne ne suffit pas à déterminer, en matière d'exercice vétérinaire, le cadre de l'activité libérale dans ces zones. En effet, la moyenne montagne reste très rurale, avec des densités en habitants très faibles (26 habitants $/ \mathrm{km}^{2}$ en montagne contre 112 habitants $/ \mathrm{km}^{2}$ sur l'ensemble du territoire métropolitain; source INSEE), mais permanente sur l'année, tandis que la haute montagne est déser- tée l'hiver au profit des vallées. Cette typologie des montagnes distingue ainsi les montagnes « urbanisées », les moyennes montagnes « agricoles ou industrialisées 》 et les hautes montagnes « résidentielles et touristiques $»$.

\section{2 / L'exercice vétérinaire de montagne}

Le vétérinaire, selon que son domicile professionnel d'exercice se situe en moyenne montagne, dans une vallée de haute montagne ou en zone urbanisée, aura donc un quotidien différent. Dans certains cas, il aura accès à la montagne toute l'année et donc aux animaux de production en altitude, tout en ayant une activité liée à la médecine et à la chirurgie des animaux de compagnie. Dans d'autres cas, l'accès à la montagne sera permis seulement en période estivale avec des animaux de rente à l'hivernage à basse altitude qui transhument sur des pâturages d'estive à la belle saison. Il faudra alors compter sur des animaux très distants de leur exploitation : le vétérinaire traitant pourra être amené, l'été, à effectuer des soins à des animaux qu'il ne suit pas le reste de l'année. Enfin, certains cabinets ou cliniques pourront avoir une activité exclusivement canine dans les zones devenues urbanisées telles que certaines villes de grande taille ( 55 communes classées en zone de montagne ont plus de 10000 habitants ; source ANEM 2013 -Association Nationale des Elus de Montagne-) ou dans des vallées de haute montagne avec une forte densité humaine. Cette activité canine est devenue essentielle pour la survie des structures vétérinaires dont l'activité rurale, elle, décroît dans ces mêmes zones.

\section{3 / Nombre de vétérinaires en montagne}

Fin 2012, en tenant compte du zonage défini par la loi Montagne, on dénombre
1543 vétérinaires exerçant en milieu de montagne (Annuaire Roy 2012). Ce nombre, comparé aux 16626 vétérinaires français inscrits au Tableau de l'Ordre des Vétérinaires (CSOV 2012b (Conseil Supérieur de l'Ordre des Vétérinaires)), correspond à peine à $10 \%$ des vétérinaires. La surface couverte par ces vétérinaires est pourtant d'environ $25 \%$ du territoire national. Le nombre de vétérinaires par habitant est également plus faible dans ces zones avec 1 vétérinaire pour 5600 habitants, comparé au reste de la France métropolitaine (1 vétérinaire pour 3700 habitants) (sources : Annuaire Roy 2013 et INSEE). En terme d'animaux et plus particulièrement pour les animaux de rente, cette différence entre plaine et montagne est la même : à titre d'exemple pour la filière bovine, 1 vétérinaire (exerçant en rurale ou mixte) pour 3300 bovins en montagne contre 1 vétérinaire (exerçant en rurale ou mixte) pour 2400 bovins en plaine (sources: Annuaire Roy 2013 et BDNI 2013 -Base de Données Nationale de l'Identification-). Cette inégalité atteint son paroxysme dans des départements de montagne où l'élevage est très présent. Ainsi, dans le Cantal, le ratio est de 1 vétérinaire pour plus de 6000 bovins (sources : Annuaire Roy 2013, février 2012). Ces données démontrent les inégalités des éleveurs devant l'accès aux soins pour leurs animaux entre montagne et plaine, même si cette difficulté d'accès au vétérinaire peut aussi être présente dans certaines zones de plaine avec une faible densité d'élevage telles qu'en périphérie des villes.

\section{4 / Caractéristiques de l'entre- prise vétérinaire de montagne}

a) Taille des structures

Les vétérinaires praticiens de montagne exercent, comme partout en France, soit en association, soit seuls. Le personnel 
Carte 1. Zones de montagne (en marron), France métropolitaine et DOM-TOM. Source : DATAR / DGCL / MAAPRAT, 31 décembre 2012.

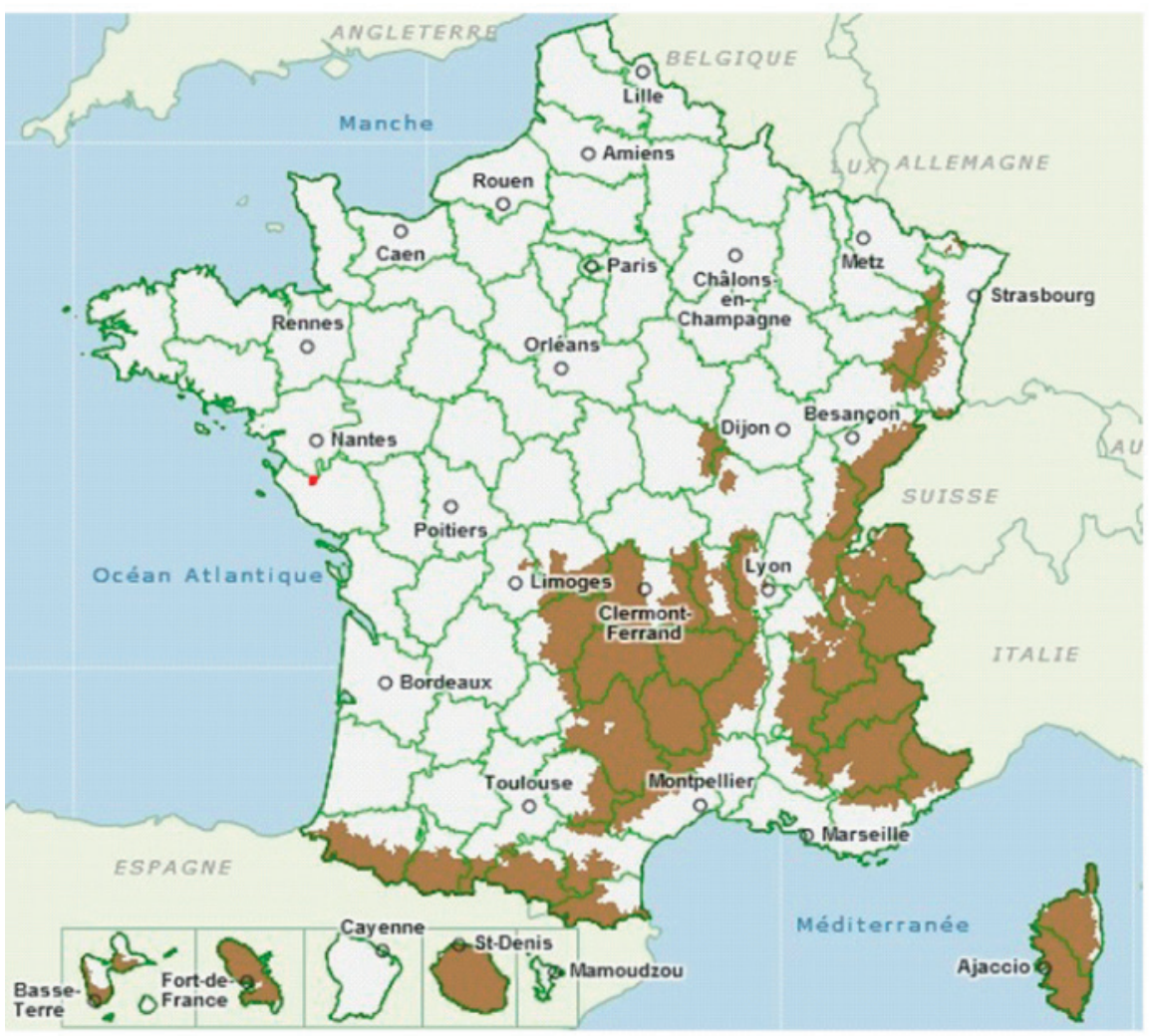

salarié est composé d'ASV (Assistante Spécialisée Vétérinaire) et éventuellement de vétérinaires (cf. § 1.4b). Leur nombre peut varier selon les années et le contexte de l'entreprise, contrairement au nombre d'associés généralement plus stable dans le temps. Fin 2012, 460 cabinets et cliniques regroupent 857 vétérinaires exerçant seuls ou associés, soit 1,86 associé par structure, à comparer à la moyenne nationale de 1,60 associé par structure (février 2012). Si l'on considère le nombre d'associés, la taille moyenne des entreprises vétérinaires de montagne est donc légèrement plus élevée que celle observée sur l'ensemble du territoire français. Cette différence peut s'expliquer, d'une part, par une offre de service vétérinaire en montagne au moins égale aux autres zones (soins aux animaux de compagnie et aux animaux de production) et, d'autre part, par des temps de parcours plus longs obligeant les structures à augmenter les moyens humains. Les praticiens exerçant seuls y sont peu nombreux, même si leur nombre n'est pas négligeable et leur importance capitale dans le maintien du maillage sanitaire territorial.

\section{b) Importance du salariat}

Outre le nombre plus élevé d'associés par structure, une bonne part des vétérinaires de montagne est salariée des entreprises (44\% ; tableau 1 - pour infor- mation : en moyenne en France, la proportion de vétérinaires salariés est de $28 \%$ (OMPL, 2010)). Ainsi, le nombre de diplômes vétérinaires par entreprise est supérieur en montagne $(3,35)$ par rapport au reste du territoire métropolitain $(2,07)$ (source : Annuaire Roy 2013). De même, si l'on compare uniquement les entreprises avec salariés (en excluant donc de la comparaison les confrères exerçant seuls), les structures de montagne ont plus de salariés en moyenne que celles de plaine (respectivement 4,01 diplômes vétérinaires en montagne contre 2,58 en plaine) (source : Annuaire Roy 2013). Cette forte proportion correspond à une augmentation saisonnière d'activités rurale (saison des vêlages) et canine (saisons touristiques) compensée par des recrutements à durée déterminée. Le salariat est, traditionnellement, une étape préalable à l'installation car il permet aux jeunes diplômés d'acquérir une expérience professionnelle. Pour les employeurs, il a aussi pour objectif de séduire des jeunes vétérinaires susceptibles de devenir associés. Malheureuse- ment, bon nombre de jeunes confrères et consœurs préfèrent aujourd'hui conserver le statut de salarié ou s'installer en zone de plaine. Le recrutement de jeunes vétérinaires revêt donc une importance capitale pour l'entreprise de montagne afin de trouver un jeune compétent et motivé pour lequel des espoirs d'association sont parfois sous-jacents.

\section{c) Féminisation de la profession}

Depuis plusieurs années, les écoles nationales vétérinaires constatent qu'une grande part des admis sont en fait des « admises » : $83 \%$ des jeunes diplômés en 2011 sont des femmes (CSOV 2012a). Ce déséquilibre devrait modifier, à terme, le ratio homme-femme vétérinaire. Dans les zones de montagne, même si les vétérinaires au féminin font leur apparition, la profession est encore majoritairement masculine : $56 \%$ des vétérinaires exerçant en montagne sont des hommes en 2012. Par ailleurs, le salariat semble préféré par les consœurs puisque les vétérinaires femmes et salariées représentent près de $30 \%$ des inscrits au tableau de l'ordre en zone de montagne (contre seulement $15 \%$ de femmes avec le statut d'associé). Les avantages sociaux du salariat, notamment en période de maternité, expliquent sans doute une partie de ce choix. Les vétérinaires hommes et associés représentent, dans le même temps, $33 \%$ de cette population $(22 \%$ sont hommes et salariés). La féminisation de la profession devrait donc accentuer le moindre attrait pour l'association. En outre, les consœurs nouvellement diplômées font généralement le choix de la canine plutôt que des productions animales $(62 \%$ en canine contre $11 \%$ en productions animales pour les diplômées 2012 ; pour information, les jeunes confrères diplômés en 2012 sont deux fois plus nombreux à préférer les animaux de production avec $20 \%$; CSOV 2013). Cette préférence pour les animaux de compagnie accompagne, pour le moment, l'évolution des structures de montagne vers une meilleure prise en charge des chiens et des chats. Cependant, le déficit constaté annuellement en vétérinaires qui se destinent à la médecine des animaux de rente (solde négatif de 97 vétérinaires en 2012, par exemple ; CSOV 2013) devrait être aggravé par cette féminisation et pourrait, à moyen terme, poser problème aux entreprises de montagne.

Tableau 1. Statistiques descriptives des vétérinaires exerçant en zone de montagne. Source : Annuaire Roy 2013.

\begin{tabular}{|l|c|c|}
\hline Nombre de vétérinaires praticiens & $\mathbf{1 5 4 3}$ & $\%$ \\
\hline libéraux & 857 & 56 \\
\hline $\begin{array}{l}\text { assistants, remplaçants, } \\
\text { collaborateurs libéraux, itinérants }\end{array}$ & 686 & 44 \\
\hline
\end{tabular}




\section{5 / Evolution de la démographie vétérinaire depuis 30 ans, I'exem- ple du Cantal}

La loi Montagne date de 1985. Il serait intéressant de comparer l'évolution, depuis cette date, de la population vétérinaire. Malheureusement, très peu de données permettent de réaliser ces statistiques : soit ces données ne sont pas disponibles, soit elles se présentent sous une forme inexploitable, soit elles sont protégées. Un observatoire national de la démographie vétérinaire, encore au stade du projet, devrait naître dans les prochaines années (CSOV, communication personnelle).

Le Cantal est un des rares départements entièrement classé en zone de montagne selon la loi Montagne (seuls la Lozère et les Hautes-Alpes présentent la même caractéristique - carte 1). Il est, à ce titre, un bon exemple. Sa population est en constante diminution depuis 1840 tandis que son cheptel bovin est, lui, en constante augmentation (174 346 bovins adultes en 1988 pour 243875 en 2000 ; Raboisson 2004). En 1980, on dénombrait 28 entreprises regroupant 59 vétérinaires diplômés. En 2011, le nombre de structures n'avait pratiquement pas évolué puisque 27 cabinets et cliniques étaient en activité mais le nombre de vétérinaires atteignait 87 (février 2012), soit 3,22 vétérinaires par cabinet. Entre ces deux dates, même si le nombre total de structures a peu évolué, leur taille s'est donc largement modifiée : baisse du nombre de cabinets avec un seul vétérinaire et augmentation du nombre d'associés dans les cabinets de groupe (février 2012). Le nombre total de vétérinaires a donc fortement augmenté, en particulier le nombre de salariés (de 6 en 1980 à 28 en 2011 ; février 2012). Cette augmentation est particulièrement marquée dans les structures ayant développé leur activité canine (février 2012).

A partir de cet exemple et en tenant compte des données citées précédemment, il semble probable que l'évolution constatée dans le Cantal soit applicable aux autres zones de montagne.

\section{2 / Quelle place pour le vété- rinaire en montagne aujour- d'hui ?}

La pratique de la médecine vétérinaire en zone de montagne est évidemment la même que celle de plaine, en tenant compte toutefois des contraintes liées à la géographie. Les vétérinaires de montagne soignent donc des animaux de rente et des animaux de compagnie même si certaines structures font parfois un choix entre ces deux activités.

\section{1 / Le vétérinaire canin en zone de montagne}

Les cabinets et cliniques de montagne exclusivement canins offrent les mêmes services que partout en France. Ces structures sont parfois présentes au cœur de vallées devenues très touristiques ou dans les grandes villes. Elles ont fait le choix d'un exercice canin exclusif, souvent pour des raisons de rentabilité et d'organisation, le maintien d'un service de soins pour quelques éleveurs étant devenu ingérable. Elles servent parfois de structures référentes en médecine et chirurgie canine auxquelles les confrères ruraux locaux font appel. Parmi les huit départements français les plus montagnards hors Corse (départements 04-05-12-15-43-48-73-74), le tiers des vétérinaires praticiens déclare avoir une activité canine exclusive (données Annuaire Roy 2013). Cette activité a donc pris une importance remarquable au sein des entreprises de montagne. Les vétérinaires exerçant en clientèle mixte à dominante canine proposent également au sein de leur structure une offre de service large : le nombre parfois important de diplômés par groupe vétérinaire permet une répartition des compétences autorisant, y compris en canine, soit à recruter de jeunes confrères canins, soit à acquérir des compétences complémentaires dans le cadre de la formation continue. De la même manière, pour les vétérinaires exerçant en clientèle mixte à dominante rurale, la canine n'est jamais abandonnée comme elle pouvait l'être autrefois (Combelles 2002) car source d'un potentiel de développement et de stabilité (face aux crises agricoles) à ne plus négliger aujourd'hui.

\section{2 / La pratique rurale en mon- tagne}

Parmi les missions du vétérinaire, il faut distinguer celles qui relèvent $\mathrm{du}$ vétérinaire traitant (médecine et chirurgie des animaux) de celles qui dépendent de son mandat sanitaire (prophylaxies collectives, application des mesures de police sanitaire).

\section{a) Des spécificités locales}

Les mutations de l'élevage en montagne ont naturellement modifié le travail du vétérinaire rural. A titre d'exemple, la transhumance a profondément changé : tandis que l'on voyait autrefois dans les montagnes, les animaux présents et nés localement avec des déplacements limités d'animaux dans l'espace, on constate maintenant la présence d'animaux d'origines géographiques, de statuts sanitaires, de races, complètement différents. Le vétérinaire traitant est généralement celui qui est sur place, ce qui n'est pas sans poser parfois quelques difficultés (respect de la règlementation sur la délivrance du médicament vétérinaire, méconnaissance du statut sanitaire et vaccinal du troupeau, moindre confiance entre l'éleveur et le vétérinaire traitant). Les dominantes pathologiques que rencontre le praticien présentent elles aussi certaines spécificités (Alzieu et al 2014) et les risques infectieux ne sont pas forcément équivalents à ceux rencontrés quelques décennies plus tôt. De même, la fabrication fromagère qui avait lieu autrefois au sein même des montagnes pendant l'estive est devenue rarissime. Le vétérinaire est maintenant amené à faire ses visites de traite ou de fabrication fromagère dans la vallée, là où se situe aujourd'hui le laboratoire de fabrication. Restent malgré tout quelques spécificités montagnardes telles que les salles de traite amovibles, placées sur le lieu même de la traite au cœur des montagnes. Elles restent, comme autrefois, le lieu de contention des animaux malades qu'il faudra examiner ou parer.

b) Des exploitations agricoles en grand nombre, des systèmes de production variés

L'élevage en montagne présente de nombreux atouts et spécificités qui influencent l'activité du vétérinaire traitant. Le nombre d'élevages en zone de montagne est encore très important : $20 \%$ des exploitations françaises et $40 \%$ des exploitations laitières sont regroupées sur les zones de montagne (Chatellier et Delattre 2003, Dervillé et Allaire 2014). Ces exploitations sont, en moyenne, de plus petite taille que pour le reste du territoire (en terme d'UGB (Unité Gros Bovin) ou de quotas laitiers; Chatellier et Delattre 2003) mais évoluent constamment vers l'agrandissement (Dervillé et Allaire 2014) et leur importance sociale et humaine n'est pas négligeable (Agreste 2011). La montagne regroupe tous les systèmes de productions (bovins lait, bovins viande, ovins lait, ovins viande, caprins lait...), même si certains massifs sont plus spécialisés dans un système que dans un autre. L'activité rurale reste donc très importante pour la plupart des cabinets vétérinaires de montagne qui ont fait le choix de maintenir cette offre de service. De plus, ces différents systèmes de production, sans omettre le traditionnel élevage fermier familial, rendent l'activité très variée.

c) Des éleveurs modernes et traditionnels à la fois

Le niveau technique des exploitants ne cesse de progresser, comme leurs moyens de travail et leur niveau de formation (Agreste 2012), y compris en santé des animaux. Le vétérinaire rural 
de montagne doit donc, à l'instar des autres grandes zones d'élevage de plaine, s'adapter à une demande nouvelle et plus technique, tout en gardant à l'esprit les spécificités de sa région. En montagne, peut-être plus qu'ailleurs, l'éleveur est attaché à ses animaux : le praticien en est le témoin au quotidien lorsque, par exemple, il aborde le thème de l'euthanasie, lorsqu' on lui demande d'effectuer des soins sans contraintes économiques ou lorsqu'il constate la surveillance toute particulière de l'éleveur pour les parturientes et les animaux nouveau-nés. L'âge parfois élevé des animaux en est un autre exemple permettant l'exercice (assez rare ailleurs) d'une médecine gériatrique (certaines pathologies, à l'instar de la médecine humaine, apparaissent plutôt chez les animaux âgés -arthrose, insuffisance rénale, tumeurs...-). La pratique vétérinaire trouve donc ici une médecine qui associe encore l'humain et l'animal, avec ce qu'elle a de plus traditionnel mais aussi de moderne.

\section{d) Des systèmes souvent spécifiques}

En élevage laitier, le « système breton » est régulièrement mis en avant et sert souvent de référence pour les publications scientifiques ou les congrès professionnels. Il n'est pourtant pas toujours adapté aux régions de montagne. En effet, les besoins sont différents (à titre d'exemple, l'alimentation à base d'ensilage de maïs n'est pas toujours possible en montagne voire proscrite pour certains cahiers des charges d'AOP (Appellation d'Origine Protégée) fromagères) et les exigences peuvent varier (la qualité du lait doit parfois tenir compte des productions de fromages au lait cru). Pourtant, le mode d'élevage en montagne évolue aussi. Le praticien doit donc faire preuve d'un esprit critique dans sa formation continue pour ne retenir que ce qu'il juge utile pour sa pratique quotidienne.

La production fromagère est commune à bon nombre de massifs montagneux : 27 fromages AOP de brebis, chèvre ou vache sont produits en zone de montagne en France (ANEM 2012) et 18 des 29 fromages AOP sont issus des montagnes françaises (Chatellier et Delattre 2003, Dervillé et Allaire 2014). Le vétérinaire peut jouer un rôle essentiel dans le suivi sanitaire des producteurs, dans le cadre de visites de traite ou de visites de fabrication. Son abord global de l'élevage est un atout précieux lors d'accidents sanitaires (salmonelloses, listérioses, toxines staphylococciques...). Une très bonne connaissance des techniques de fabrication, des cahiers de charges et des filières est indispensable pour adapter son conseil à chaque situation de crise. Certains vétérinaires sont devenus de véritables référents en la matière.
L'élevage des chevaux de trait est un autre exemple de production très présente en montagne. Le Cantal est, par exemple, le premier département français pour la filière " chevaux lourds ». La médecine équine de montagne doit donc prendre en compte une dimension sanitaire et économique, moins présente pour les chevaux de selle.

\section{3 / Le vétérinaire sanitaire en zone de montagne}

\section{a) Un maillage qui a fait ses preuves}

Le maillage sanitaire français s'appuie sur un réseau de vétérinaires régulièrement répartis sur le territoire national, montagnes comprises. Cette présence de professionnels de la santé animale a pour mission, par le biais de leur habilitation ou mandatement, de gérer d'éventuels foyers de maladies animales, de réaliser des visites sanitaires obligatoires et d'assurer une surveillance épidémiologique de maladies émergentes. La gestion des différentes épizooties des dernières décennies (Encéphalopathie Spongiforme Bovine, Fièvre Aphteuse, Grippe Aviaire, Fièvre Catarrhale Ovine -FCO- ), qui s'appuie sur les interventions des vétérinaires sanitaires, a prouvé l'efficacité du dispositif. Ainsi, le cheptel français est officiellement indemne, à ce jour, de tuberculose, de fièvre aphteuse, de brucellose, de FCO, de varron. Parmi ces affections, certaines sont des zoonoses (maladies animales transmissibles à l'Homme).

Les zones de montagne constituent des entités épidémiologiques très importantes car elles présentent des facteurs de risque particuliers : estives et pâturages collectifs, contacts fréquents des animaux avec la faune sauvage, transhumances d'animaux provenant de régions différentes, n'en sont que quelques exemples. Ici donc, plus qu'ailleurs, la veille sanitaire doit être attentive. Ainsi, pour certaines maladies non règlementées comme l'IBR (Rhinotrachéite Infectieuse Bovine), le nombre d'animaux encore séropositifs est important dans la majorité des zones de montagne. En effet, les conditions et les facteurs de risque décrits plus haut rendent la tâche plus difficile. Au contraire de ce qui est fait jusqu'ici, ce sont donc ces zones qui mériteraient une accentuation des efforts en matière de sanitaire et donc un plus grand soutien du réseau vétérinaire : tandis que l'Etat reconnaît les spécificités de l'élevage de montagne (par exemple par les subventions spécifiques qui lui sont allouées), l'exercice du mandat sanitaire est considéré comme équivalent entre plaine et montagne. Enfin, le schéma classique de gestion des maladies animales par les organisations professionnelles agricoles n'obtient pas, en montagne, d'aussi bons résultats qu'en plaine : les plans sanitaires se heurtent souvent aux réalités du terrain et les décisions décentralisées ne sont pas toujours adaptées.

\section{b) Mais qui atteint ses limites}

Comme vu précédemment, l'évolution démographique vétérinaire n'est pas en faveur d'un meilleur maillage rural et le réseau vétérinaire de montagne exerçant en animaux de production pourrait, à court terme, montrer des carences. A titre d'exemple, si l'on compare deux départements dont le cheptel bovin est équivalent en nombre de têtes, l'un de plaine (l'Orne) et l'autre de montagne (le Cantal), la « zone d'influence » d'un cabinet vétérinaire est complètement différente (carte 2). La densité du maillage vétérinaire y est clairement en faveur du département de plaine. La gestion de la dernière épizootie (FCO, 2007-2012) a mis en évidence les limites du système : le manque de reconnaissance des pouvoirs publics, les pressions des organisations sanitaires agricoles et le manque de clarté dans la stratégie de lutte, ont nui au réseau sanitaire dans son ensemble (février 2012). Certains vétérinaires ont choisi de cesser leur activité, d'autres ont préféré ne pas obéir aux consignes (février 2012). Finalement, même si les vétérinaires sont parvenus à accomplir cette mission en vaccinant des millions d'animaux en un temps record, permettant ainsi à la France de retrouver son statut indemne dès 2012, un goût amer persiste dans les relations du tryptique sanitaire français «EleveursVétérinaires-Etat ». Cet épisode doit servir d'avertissement aux autorités pour une meilleure considération du réseau sanitaire formé par les vétérinaires, en particulier dans les zones où celui-ci est le plus fragile.

\section{c) Un lien fragile à préserver}

$\mathrm{Au}$ cœur du réseau, le lien entre les vétérinaires et leur $\mathrm{DD}(\mathrm{CS}) \mathrm{PP}$ (Direction Départementale de la Cohésion Sociale et de la Protection des Populations ancienne Direction des Services Vétérinaires) est essentiel. La proximité des vétérinaires de terrain avec leurs éleveurs permet d'aider les Services de l'Etat en l'éclairant sur des contextes particuliers. C'est le cas en montagne peut-être plus qu'ailleurs : les bâtiments d'élevage traditionnels de montagne, par exemple, ne sont pas toujours en accord avec les recommandations et normes en vigueur, notamment en termes de bien-être (animaux à l'attache pendant plus de 6 mois par an, ouvertures de petite taille limitant l'entrée de lumière, surface au sol par animal réduite).

Enfin, la part financière représentée par les missions d'Etat (opérations de 
Carte 2. Comparaison, entre les départements du Cantal (à gauche) et de l'Orne (à droite), de la densité en cabinets et cliniques vétérinaires et de leur zone d'influence respective. Source : inventaire communal INSEE - attractivité des vétérinaires.

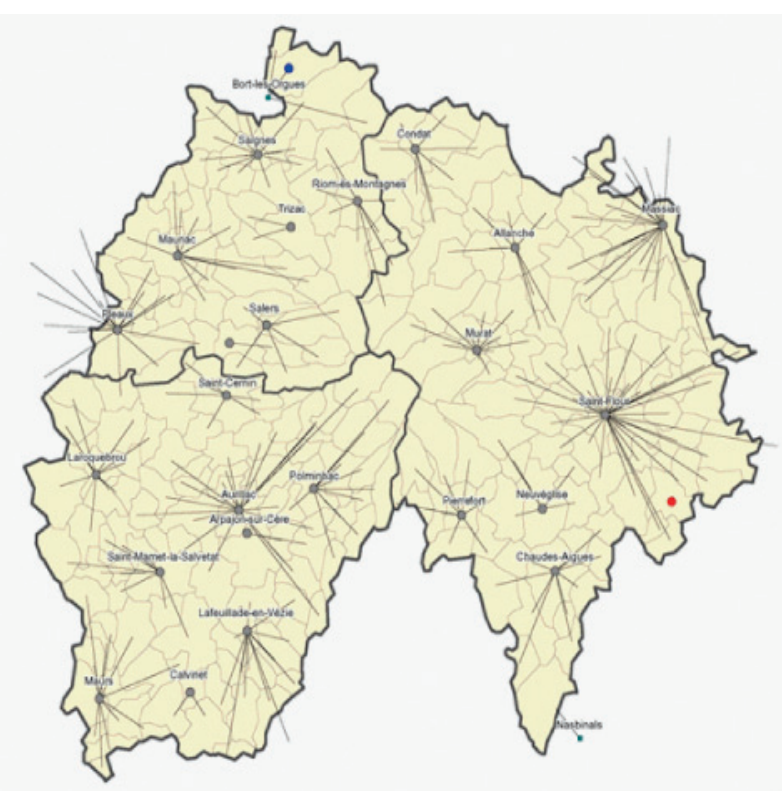

prophylaxie collective, missions de police sanitaire) est devenue minoritaire dans le revenu des vétérinaires : elle est passée de $30 \%$ en 1991 à $3 \%$ en 2006 (Guéné 2008). L'exercice du mandat sanitaire en montagne est beaucoup moins rémunérateur, proportionnellement, qu'ailleurs. En effet, les tarifs des opérations de prophylaxies collectives ne tiennent pas compte, le plus souvent, des spécificités de montagne qui compliquent le travail $\mathrm{du}$ vétérinaire (absence fréquente de moyens de contention, existence de multiples sites d'élevage pour un même exploitant, distances à parcourir et temps de parcours supérieurs). Certaines structures vétérinaires ont fait le choix, en zones dites de « haute et moyenne montagne résidentielles et touristiques $»$, de ne plus assurer les soins aux grands animaux, en grande partie pour des raisons de manque de rentabilité horaire (temps de déplacement trop longs) : ces zones constituent maintenant des zones blanches, sans réseau sanitaire de proximité. Ainsi et malheureusement, il est aujourd'hui plus fréquent de rencontrer, en particulier en haute montagne, des éleveurs dépourvus de vétérinaire sanitaire.

\section{3 / Vétérinaire à la monta- gne : une profession désuète ou promise à un avenir ensoleillé ?}

\section{1 / La « canine " pour tous ?}

Dans le cas des zones de montagne très urbanisées ou touristiques, les entreprises n'offrent la plupart du temps qu'un service de soins pour animaux de compagnie : cette tendance évolue maintenant vers la périphérie des grandes villes, le suivi d'un nombre restreint d'élevages étant devenu trop difficile à organiser. Dans le cas des zones d'élevage, si la médicalisation des animaux de compagnie permet de faire progresser encore les entreprises vétérinaires rurales, elle ne pourra pas suffire, à elle seule, à justifier la taille des structures vétérinaires de montagne. En effet, les cabinets vétérinaires de montagne sont encore, en grande majorité et malgré tout, largement dépendant de leur activité en productions animales. En outre, le besoin en soins vétérinaires est relié à la densité animale, très élevée pour les animaux de production comme vu précédemment. Il faudra donc et malgré tout continuer à assurer un service à domicile performant et continu.

\section{2 / Le vétérinaire et l'éleveur, même combat}

L'avenir du vétérinaire rural de montagne est lié à celui de l'éleveur: les zones de montagne, dont les systèmes de production sont souvent variés, semblent moins exposées aux aléas économiques par rapport aux régions très spécialisées. Néanmoins, les politiques économiques jouent un grand rôle dans l'avenir de ces zones puisque l'on sait que les aides sont essentielles au maintien de leur activité (Perret 1999, Martin et al 2014). Quels seront les effets de la nouvelle PAC (Politique Agricole Commune) 2014-2020 sur le niveau des aides pour ces régions? Le « verdissement » mis en avant (Sans 2011) serat-il un avantage? Qu'en sera-t-il de l'après-quotas? (Dervillé et Allaire 2014)

De même, si l'on veut maintenir pour l'éleveur de montagne un service de soins médicaux aux animaux, les décisions politiques prises à l'avenir ne devront pas mettre en péril l'équilibre économique des entreprises vétérinaires. Le maintien du couplage "prescriptiondélivrance " (les vétérinaires peuvent actuellement à la fois prescrire et délivrer les médicaments vétérinaires) est, à cet égard, déterminant.

\section{3 / S'adapter : une nécessité}

L'attachement de l'éleveur à ses animaux et leur valeur marchande parfois élevée maintiennent un urgentisme conséquent, même si l'éleveur, devenu infirmier de son élevage, gère dorénavant une bonne partie des soins de première intention. Outre ce service d'urgences qui devrait persister, et devant les enjeux et les attentes des éleveurs, les vétérinaires devront toujours améliorer leurs connaissances et leur offre. La compétence ne pourra suffire à elle seule aux éleveurs de demain (Brard 2010) : il faudra être rentable pour l'élevage, proposer des services innovants et agir en amont par des actions préventives afin de limiter le risque d'un accident sanitaire devenu trop difficile à gérer et dangereux pour l'exploitation. Le praticien a su jusqu'ici s'adapter, à l'instar des zones de plaine, en proposant de nouveaux services dans le cadre d'une approche technico-économique du sanitaire : formation, alimentation, suivi reproduction, audit d'élevage, bilan sanitaire, etc. Le développement des systèmes d'information (internet haut débit) permet maintenant, à la montagne comme ailleurs, d'accéder à des sources bibliographiques nouvelles, qui s'ajoutent aux publications dans des revues professionnelles et au catalogue de formation continue. Cette adaptation aux systèmes de production 
n'est pas spécifique à la montagne, mais elle y est encore plus essentielle pour participer à améliorer les performances financières des exploitations de montagne, déjà fortement pénalisées par rapport à la plaine (Martin et al 2014).

\section{4 / Pas d'avenir sans recrutement}

L'avenir des structures de montagne passe par le renouvellement des équipes soignantes : l'entreprise vétérinaire de montagne emploie un grand nombre de vétérinaires salariés qui font plutôt le choix de partir que de rester en s'associant. Les structures doivent donc régulièrement recruter de nouveaux collaborateurs pour compenser ces départs. $\mathrm{Ce}$ recrutement est parfois difficile, notamment pour les structures de petite taille, les jeunes confrères étant parfois gênés par l'organisation du travail et des loisirs. De même pour les entreprises de plus grande taille, convaincre de jeunes confrères de travailler en montagne est parfois délicat et il faut maintenant faire appel à des vétérinaires diplômés à l'étranger, plus enclin à exercer en productions animales (56\% des vétérinaires diplômés hors de France déclarent une compétence en « animaux de rente »; CSOV 2011). Toutefois, ces confrères sont encore peu nombreux et représentent moins de $3 \%$ des vétérinaires libéraux de montagne (Annuaire Roy 2013). Il semble que, pour s'installer en montagne, mieux vaut avoir fait ses études à proximité : plus des $2 / 3$ des vétérinaires de montagne sont diplômés des écoles de Lyon et de Toulouse (tableau 2). L'employeur devrait donc plutôt recruter dans ces écoles ou à l'étranger. Aujourd'hui, de nombreux facteurs devraient rendre beaucoup plus séduisant l'exercice en montagne. Ainsi, l'isolement géographique est de moins en moins ressenti (développement des réseaux routiers, autoroutiers et ferroviaires) avec un accès plus rapide aux grandes métropoles ou aux aéroports. Le regroupement des vétérinaires faci-

Tableau 2. Statistiques descriptives des vétérinaires exerçant en zone de montagne. Source : Annuaire Roy 2013.

\begin{tabular}{|l|c|c|}
\hline Ecole d'origine & Nb. & $\%$ \\
\hline Lyon & 282 & 40 \\
\hline Toulouse & 185 & 26 \\
\hline Alfort & 79 & 11 \\
\hline Liège & 81 & 11 \\
\hline Nantes & 56 & 8 \\
\hline Gand & 7 & 1 \\
\hline Autres écoles (1 à 3 individus par école) & 20 & 3 \\
\hline
\end{tabular}

lite l'organisation du travail en libérant des jours de vacances, y compris en période de forte activité. L'exercice est plus varié qu'ailleurs avec une pathologie assez riche et le vétérinaire de montagne garde, en général, un contact avec de nombreuses espèces dans sa pratique quotidienne. La qualité de l'environnement est maintenant recherchée par tous et la montagne rassemble ces conditions. Enfin, les caractéristiques de l'élevage en montagne et les rapports humains sont autant de facteurs séduisants pour le vétérinaire rural. La pyramide des âges de ces vétérinaires atteste d'ailleurs de cet engouement persistant pour la montagne (chaque décennie, entre 1980 et 2010, rassemble $30 \%$ de confrères - Annuaire Roy 2013), signe d'un renouvellement réel des vétérinaires en montagne, jusqu'à présent.

\section{Conclusion}

La montagne est séduisante et l'exercice vétérinaire y est tout autant. Mais la montagne souffre de handicaps pesant parfois lourd dans la balance à l'heure du choix de l'installation (moindre attrait des jeunes diplômés pour les productions animales, contraintes climatiques et géographiques...) et le jeune vétéri- naire finalement installé en montagne est celui qui mettra au premier plan l'amour du métier. Ainsi, l'accès aux soins des animaux en milieu de montagne, à l'instar des autres professions médicales, est devenu plus difficile qu'autrefois même si les vétérinaires, regroupés en unités de compétences, sont encore présents et proposent un service adapté comparable aux zones de plaine. L'importance de ces structures dans la réussite du modèle sanitaire français est considérable. Les pouvoirs publics doivent en prendre conscience rapidement, faute de quoi la pérennité du maillage vétérinaire et la surveillance épidémiologique des maladies animales seront orphelines dans certaines zones de montagne. Les spécificités de l'exercice vétérinaire en montagne doivent être mieux prises en considération, à l'instar des actions en faveur de l'élevage dans ces zones, pour inverser la tendance et inciter les vétérinaires à s'y installer. L'Ordre des Médecins et certaines collectivités locales ont déjà pris, depuis plusieurs années, des mesures pour encourager et aider les médecins à faire le choix de ces zones déshéritées. De telles incitations tenant compte des contraintes professionnelles en montagne pourraient également être appropriées pour la profession vétérinaire en zone de montagne.

\section{Références}

Agreste, 2011. Recensement agricole 2010, structure des exploitations agricoles : les productions se concentrent dans les exploitations spécialisées. Agreste Primeur, $\mathrm{n}^{\circ} 272,4 \mathrm{p}$.

Agreste, 2012. Recensement agricole 2010, formation des chefs d'exploitation: jeunes agriculteurs, parmi les actifs les mieux formés. Agreste Primeur, $\mathrm{n}^{\circ} 281,4 \mathrm{p}$.

Alzieu J.P., Brugère-Picoux J., Brard C., 2014. Particularités pathologiques des ruminants domestiques en estive dans les montagnes françaises. In : Spécificités de l'élevage de ruminants en montagne. Grosclaude J., Thibier M., Baumont R. (Eds). Dossier, INRA Prod. Anim., 27, 31-40.

ANEM, 2012. Le dossier de l'Elu de montagne. http://www.anem.org/upload/pdf/Le classe-

\section{ment_Montagne $06 \_2012$ 20120601101727.pdf}

Brard C., 2010. Avenir de l'entreprise vétérinaire en productions animales, en France. Bull. Acad. Vét. France, 163, 307-312.

Chatellier V., Delattre F., 2003. La production laitière dans les montagnes françaises : une dynamique particulière pour les Alpes du Nord. INRA Prod. Anim., 16, 61-76.

Combelles C., 2002. Chroniques d'un vétérinaire rural au cours du $X^{\text {ème }}$ siècle dans le Cantal. Thèse de Doctorat Vétérinaire, Alfort, France, $107 \mathrm{p}$.

CSOV, 2011. Vous avez dit numerus clausus! La revue de l'Ordre des Vétérinaires, 45, 2627. http://www.veterinaire.fr/img/upload/0/0/ 0/318 Stats\%20ROV\%2045.pdf
CSOV, 2012a. Démographie professionnelle en France et en Allemagne: quelques éléments de comparaison. La revue de l'Ordre des Vétérinaires, 47, 26-27. http://www.veterinaire.fr/ img/upload/0/0/0/313 Stats\%20ROV\%2047.pdf

CSOV, 2012b. Démographie professionnelle : focus sur les nouveaux inscrits. La revue de l'Ordre des Vétérinaires, 48, 24-25. http:// www.veterinaire.fr/img/upload/0/0/0/314 stats \%20ROV\%2048.pdf

CSOV, 2013. Evolution de la démographie professionnelle en 2012. La revue de l'Ordre des Vétérinaires, 49, 24-25. http://www.veterinaire.fr/img/upload/0/0/0/315 Stats $\% 20$ ROV\%2 049.pdf

Dervillé M., Allaire G., 2014. Quelles perspectives pour les filières laitières de montagne 
après la suppression des quotas laitiers ? Une approche en termes de régime de concurrence. In : Spécificités de l'élevage de ruminants en montagne. Grosclaude J., Thibier M., Baumont R. (Eds). Dossier, INRA Prod. Anim., 27, 1730 .

Février E., 2012. La profession vétérinaire dans le département du Cantal : évolution des structures au cours des 30 dernières années. Bull. Acad. Vét. France, 165, 73-80.

Guené C., 2008. Vers une profession vétérinaire du XXI ${ }^{\text {ème }}$ siècle. Rapport au Premier Ministre et au Ministre de l'Agriculture et de la Pêche. http://agriculture.gouv.fr/IMG/pdf/rapport-definitif-221208-.pdf
Martin B., Lherm M., Béranger C., 2014. Evolutions et perspectives de l'élevage des ruminants dans les montagnes françaises. In : Spécificités de l'élevage de ruminants en montagne. Grosclaude J., Thibier M., Baumont R. (Eds). Dossier, INRA Prod. Anim., 27, 5-16.

OMPL, 2010. Portrait statistique et prospectif des cabinets vétérinaires. Téléchargeable au http://www.observatoire-metiers-entreprisesliberales.fr/fichiers utilisateurs/fichiers/statistiques/ETUDES/ETUDES\%20THEMATIQUES/SANTE/VETO/2010/portrait branch e cabinets veterinaires octobre 2010.pdf?PH PSESSID $=\mathrm{d} 7 \mathrm{~b} 631 \mathrm{a} 39 \mathrm{ad} 42 \mathrm{e} 334269 \mathrm{e} 606 \mathrm{cc} 7 \mathrm{aa}$ $\underline{\mathrm{c} 32}$
Perret E., Thomson E., Dobremez L., Chantry E., 1999. Pour tous les systèmes d'élevage en montagne, les subventions sont essentielles au maintien de l'activité. Les cahiers de l'Agreste, 46, 23-34.

Raboisson D., 2004. Evolution raciale du cheptel bovin français des années 1970 aux années 2000 : analyse à partir des données des Recensements Généraux agricoles de 1979, 1988 et 2000. Thèse de Doctorat Vétérinaire, Toulouse, France, 173p.

Sans P., 2011. Vers une politique agricole commune plus verte et plus juste ? Le Nouveau Praticien Vétérinaire, 4, 63-66.

\title{
Résumé
}

En France, les vétérinaires praticiens bien qu'exerçant tous la même profession n'exercent pas tous le même métier : on peut opposer notamment les vétérinaires des villes à ceux des campagnes. Pour ces derniers, des différences apparaissent aussi selon leur domicile professionnel. En montagne notamment, il existe des contraintes particulières pour l'exercice de la médecine et de la chirurgie des animaux qui sont décrites dans cet article. Ces zones sont victimes d'une intense désertification qui concerne aussi et malheureusement les vétérinaires. En réponse à cette situation, les vétérinaires ont dû s'adapter pour maintenir une offre de service aussi efficace et performante qu'en ville ou qu'en zone de plaine pour les éleveurs et les propriétaires d'animaux de compagnie. Le recrutement de jeunes vétérinaires est devenu essentiel à la survie des entreprises vétérinaires et donc pour le réseau sanitaire. En effet, la veille sanitaire permise par ces structures est très importante en montagne, sans doute plus qu'ailleurs étant donné les risques sanitaires liés à l'élevage dans ces régions. Les éleveurs, devenus très performants ici comme en plaine, exigent les meilleurs soins et conseils de la part de leur vétérinaire traitant. Mais l'implication du vétérinaire de montagne va bien souvent au-delà d'un simple contrat de soin et nécessite de sa part un investissement personnel particulier.

\begin{abstract}
Function of the veterinarian in the mountain areas: evolution and future

In France, veterinarians in practice, even though they all exercise the same profession, may display various and distinct kinds of jobs: vets in cities vs. vets in the countryside, taking care of livestock or pets etc. Moreover even in the rural area, there are striking differences of approach depending on the region or area of practice. This is particularly the case for mountainous areas which as a result, generates various constraints for the practice of medicine and surgery of animals that are described in this presentation. In addition, these zones show an intense desertification that consequently and dramatically impacts vet practices. In response to such a challenge, the vets have to adapt themselves and find the most appropriate ways to maintain local services to the farmers in as an efficient manner as for pets in towns. The recruitment of young veterinarians becomes critical for the survival of rural vet practices and thus for the animal health surveillance veterinary network as a whole but more particularly in mountainous areas given their specific sanitary risks. Farmers in such mountainous regions have become economically successful during recent decades similarly to what is noticed in plains. They too require great professionalism and efficiency from their vets both in terms of farming strategy and prevention and cure of diseases. In addition, the implication of the vet in such areas is often well beyond a simple contract and practically requires quite a personal investment.
\end{abstract}

ROY C., 2014. La place du vétérinaire en milieu de montagne : évolution et futur. In : Spécificités de l'élevage de ruminants en montagne. Grosclaude J., Thibier M., Baumont R. (Eds). Dossier, INRA Prod. Anim., 27, 41-48. 